\title{
Über Höhensonnenwirkung nach Röntgenbestrahlung bei Psoriasis.
}

\author{
Von \\ San.-Rat Dr. Baer (Frankfurt a. M.). \\ (Eingegangen am 24. Februar 1921.)
}

Zwei Fälle von Psoriasis, die in ihrem Verlaufe eine gewisse Übereinstimmung zeigen und auch vom therapeutischen Gesichtspunkte aus merkwürdig sind, bieten mir Anla $B$, sie zu veröffentlichen.

Fall 1. 51/2jähriges Kind. Dasselbe wurde mir zum erstenmal vor 2 Jahren gebracht und ich stellte damals eine unbedeutende, an den Knien und auf den Glutaeen lokalisierte Psoriasis fest, für die ich Salben verordnete.

Seit dieser Zeit sah ich die kleine Patientin nicht wieder. Erst am 9. VIII. 1920 wurde sie mir wieder vorgestellt mit den Erscheinungen einer diffusen, über den ganzen Körper disseminierten Psoriasis; nur Handteller und Fußsohlen waren frei, die einzelnen Plaques wiesen starke Infiltration auf in einer Weise, wie man sie bei Kindern dieses Alters selten sieht.

Als Ursache dieses starken Ausbruches war eine von anderer Seite ausgeführte Röntgenbehandlung mit großer Wahrscheinlichkeit anzusehen; zwar sollen die vorher spärlich vorhanden gewesenen Plaques nach der Röntgenbehandlung zurückgegangen sein, doch sei kurz nach der ziemlich ausgedehnten Röntgenbestrahlung (über die Dosierung war nichts zu erfahren) die Erkrankung in der obenbeschriebenen Weise ausgebrochen.

Tch behandelte etwa 2 Monate lang mit den üblichen antipsoriatischen Mitteln, doch ohne Erfolg. Es trat zwar eine Abflachung der einzelnen psoriatischen Efflorescenzen ein, doch erwies sich die endgültige Beseitigung als außerordentlich schwer. Nun entschloß ich mich zur Höhensonnenbehandlung; in Abständen von 3 Tagen wurde der Körper mit Fernbestrahlungen behandelt und zwar so, daB in einer Entfernung von 50, später 40 und $30 \mathrm{~cm}$ von vorn und hinten je 5 Minuten lang bestrahlt wurde.

Der Erfolg war verblüffelnd; nach 6 Bestrahlungen war die Hautkrankheit restlos verschwunden.

Fall 2. 37jähriger Beamter, leidet seit 1903 an Psoriasis. Oktober 1919 Verschlimmerung der Erkrankung. Er konsultierte einen Spezialisten, der mit ihm eine Röntgenbehandlung vornahm, und zwar wurden die Handrücken, Oberand Unterarme, Becken, Ober- und Unterschenkel und die Ohrmuscheln bestrahlt.

Während der Röntgenbehandlung fielen die um die Ohrmuscheln befindlichen Kopfhaare aus. Kurz nach Beendigung der Behandlung trat an beiden Händen eine starke Entzündung auf. Neue Herde auf dem Rücken, am rechten Oberschenkel, in der Beckengegend, an beiden Unterschenkeln, am rechten Oberschenkel bildeten sich, die so schmerzhaft waren, daB er 4 Wochen lang das Bett hüten mußte. 
Am 5. VII. 1920 sah ich den Patienten zum erstenmal mit einer mächtigen Psoriasis, der ganze Rücken war lückenlos von einem stark infiltrierenden psoriatischen Herd bedeckt. Außerdem waren an Oberarmen und unterhalb beider Knie psoriatische Herde wahrzunehmen. Unterhalb des rechten Knies befand sich, noch offenbar als Folge der damaligen Röntgenbestrahlung, eine noch nicht ausgeheilte handtellergroße Excoriation.

Patient war lange Zeit außerdem mit den verschiedensten antipsoriatischen und Arsenmitteln vorbehandelt worden.

Ich leitete, da mir keine andere Wahl blieb, sofort eine Behandiung mit Höhensonne ein, indem ich den Rücken in einem Abstand von $30 \mathrm{~cm}$, später $20 \mathrm{~cm} 10$ Minuten alle 3 Tage bestrahlte.

Nach 8 Bestrahlungen im Verlaufe von etwa 3 Wochen war die Psoriasis des Rückens abgeheilt und auch die nichtbestrahlt gewesenen Stellen hatten sich vollständig zurückgebildet ${ }^{\mathbf{1}}$ ).

Das gemeinsame in diesen beiden Fällen ist zunächst die Beobachtung, daß die Psoriasis nach Röntgenbehandlung in verstärktem Maße aufgetreten ist. $\mathrm{Ob}$ in dem Fall 1 überdosiert wurde, bleibe dahingestellt.

Ganz zweifellos wurde aber im Fall 2 überdosiert. Wir müssen also annehmen, daß die Röntgenbehandlung provozierend auf die Erkrankung gewirkt hat. Dies ist zum mindesten auffallend, denn es ist nicht bekannt, daß bei anderen durch Röntgenstrahlen beeinflubten Dermatosen derartige Verschlinmerungen sich gezeigt haben.

Wohl wissen wir, daß die Psoriasis auf chemische Reize hin universell werden kann, doch beobachtete ich bei meinen Fällen zum erstenmale, daß auch die Röntgenstrahlen imstande sind, provozierend auf die Psoriasis zu wirken.

Niemals aber sah ich, auch nach chemischer Reizung eine derartige Verschlimmerung der Erkrankung, wie sie im Fall 2 auftrat.

Des weiteren ist in beiden Fällen gemeinsam die Tatsache, daß die Höhensonne die Erkrankung in auffallend kurzer Zeit zur Heilung brachte. Ganz besonders im Falle 2 zeigte sich, daß die Höhensonne den üblichen antipsoriatischen Mitteln bedeutend überlegen war.

Meines Wissens sind derartige Beobachtungen nicht veröffentlicht ${ }^{2}$ ).

Daß eine vorsichtig ausgefübrte Röntgenbehandlung zumal in den Fällen, bei denen es sich um circumscripte, inveterierte Formen handelt, Vorzügliches leistet, braucht an dieser Stelle nicht besonders erwähnt zu werden.

Aber bei allen ,akuten" Formen, sowie bei Jugendlichen, ist Vorsicht geboten.

1) In der Zwischenzeit neues Rezidiv.

2) Anmerkung bei der Korrektur. In dem inzwischen ersehienenen Fandbuch der Röntgen- und Radiumtherapie (Bd. II) erwähnt Wetterer die Beobachtung, auf die zuerst $\mathrm{H}$ abeknech $\mathrm{t}$ aufnerksam gemacht hat, daß bei stärkerer Bestrahlung sich die gesunde Haut in der LTmgebung eines Erkrankungsherdes psoriatisieren kann. 
Hier käme, nach meinen Erfahrungen aber lediglich als Adjuvans, unter Umständen die Behandlung mit ultravioletten Strahlen in Betracht.

Uber die Kompressionsbehandlung fehlen mir eigene Erfahrungen.

Die Fernbestrahlung dagegen wurde von mir in vereinzelten Fällen angewandt. Sie steht der Röntgenwirkung auf jeden Fall nach, leistet aber Brauchbares überall da, wo sich aus irgend einem Grunde die Anwendung der üblichen Antipsoriatica nicht durchführen läßt.

Wichtig erscheint es mir, darauf hinzuweisen, daß, was auch von anderen Beobachtern (s. u.) betont wird, die Höhensonne fast nur dann Erfolge zeitigt, wenn sie leichte Reizwirkungen auf der Haut verursacht.

Aus der mir zugänglichen Literatur läßt sich feststellen, daß die Kompressionsbehandlung nur in den seltenen Fällen Anwendung verdient, bei welchen es sich um circumscripte, infiltrierte Plaques handelt. So sah günstige Erfolge von einer derartigen Behandlung Heyman (Dtsch. med. Wochenschr. 1907), ferner Lohde (ebenda) und Lüth (Med. Klin. 1908)..

Über günstige Wirkung der Fernbestrahlung bei Psoriasis berichtete Becker (Dtsch. med. Wochenschr. 1907). Er meint, daß es sehr wohl möglich sei, die Psoriasis auch durch Distanzbestrahlung zur Heilung zu bringen; dies hat den Vorteil, daß große Flächen belichtet werden können; einfache Hyperämie scheint ihm das Ideal der zur Heilung der Psoriasis notwendigen Reaktion zu sein.

Kromayer (Röntgen-Radium-Licht in der Dermatologie) drüokt sich über die Wirkung des Quarzlichtes bei Höhensonne sehr vorsichtig aus. Erwähnenswert und interessant ist seine Beobachtung, da $B$ auch nichtbelichtete Stellen auf die Bestrahlung reagieren können; ich habe diese Beobachtung in meinem oben zitierten Fall 2 gemacht; trotzdem nur die großen Plaques am Rücken bestrahlt wurden, gingen die Psoriasisefflorescenzen auch an den nichtbestrahlten Teilen zurück. Stümpke (Die medizinische Quarzlampe) will das Quarzlicht nur bei circumscripten, ziemlich infiltrierten Plaques angewandt wissen, die jeder anderen Therapie trotzten und zwar in Form der Kompressionsbehandlung. Die Distanzbestrahlung empfiehlt er nur für akute, diffus ausgebreitete Prozesse.

Über günstige Erfahrungen an einem großen Material berichtete Linser (Med. Klin. 1915). Er hatte die besten Erfolge, wenn die psoriatischen Stellen in leichte seröse Exsudation gerieten. Vorsichtige Behandlung unter Vermeidung einer Lichtreaktion gäbe nicht die guten Resultate. Er erwähnt dann einen Fall, den ich deswegen anführe, weil er ähnlich gelagert ist, wie meine beiden oben angeführten Fälle: bei einem älteren Mann, der früher alljährlich eine Salbenkur mit gutem Erfolge gemacht hatte, nun aber ohne bekannte Ursache das Chrysarobin nicht mehr vertrug, entstand ein heftiges universelles Erythem; da ein längerer Versuch mit indifferenten Salben und Bädern keine Besserung brachte, wurde ein vorsichtiger Versuch mit Höhensonne gemacht, der Erfolg war ein glänzender, der Patient war in 14 Tagen geheilt. Dieser Fall ist ganz analog den beiden oben beschriebenen Fällen. Nur mit dem Unterschied, daß anstatt der vorhergehenden Röntgenbestrahlung hier die Chrysarobindermatitis den günstigen Boden für die Wirkung der Höhensonne darstellte.

Nur in einem Punkte weichen meine beiden Fälle ab. Während nämlich sämtliche Autoren zur Heilung der Psoriasis durch Quarz- 
54 Baer: Über Höhensonnenwirkung nach Röntgenbestrahlung bei Psoriasis.

licht die Bildung einer mehr oder weniger starken Dermatitis verlangen, bewirkte die Höhensonnenbestrahlung die Rückbildung der Psoriasis bei den beiden vorher mit Röntgen bestrahlten Fällen ohne jede Hautreizung.

Wollte man nun versuchen, sich den Vorgang der Abheilung zu erklären, so könnte man sich vorstellen, daß der hypothetische Parasit, für den auch neuerdings Lipschütz (Arch. f. Dermatol. u. Syphilis Bd. 127) eintritt, durch die Wirkung der Röntgenbestrahlung in einen derartigen Reizzustand versetzt wurde, daß er durch die utravioletten Strahlen leicht erreicht werden konnte. 